The Astrophysical Journal, 665:785-793, 2007 August 10

(C) 2007. The American Astronomical Society. All rights reserved. Printed in U.S.A.

\title{
RETIRED A STARS AND THEIR COMPANIONS: EXOPLANETS ORBITING THREE INTERMEDIATE-MASS SUBGIANTS ${ }^{1}$
}

\author{
John Asher Johnson, ${ }^{2}$ Debra A. Fischer, ${ }^{3}$ Geoffrey W. Marcy, ${ }^{2}$ Jason T. Wright, ${ }^{2}$ Peter Driscoll, ${ }^{4}$ \\ R. Paul Butler, ${ }^{5}$ Saskia Hekker, ${ }^{6}$ Sabine Reffert, ${ }^{7}$ and Steven S. Vogt ${ }^{8}$ \\ Received 2007 February 13; accepted 2007 May 8
}

\begin{abstract}
We report precision Doppler measurements of three intermediate-mass subgiants obtained at Lick and Keck Observatories. All three stars show variability in their radial velocities consistent with planet-mass companions in Keplerian orbits. We find a planet with a minimum mass $M_{P} \sin i=2.5 M_{\mathrm{J}}$ in a 351.5 day orbit around HD 192699 , a planet with a minimum mass of $2.0 M_{\mathrm{J}}$ in a 341.1 day orbit around HD 210702, and a planet with a minimum mass of 0.61 $M_{\mathrm{J}}$ in a 297.3 day orbit around HD 175541. Mass estimates from stellar interior models indicate that all three stars were formerly A-type, main-sequence dwarfs with masses ranging from 1.65 to $1.85 M_{\odot}$. These three long-period planets would not have been detectable during their stars' main-sequence phases due to the large rotational velocities and stellar jitter exhibited by early-type dwarfs. There are now nine "retired" (evolved) A-type stars $\left(M_{*}>1.6 M_{\odot}\right)$ with known planets. All nine planets orbit at distances $a \geq 0.78 \mathrm{AU}$, which is significantly different from the semimajor axis distribution of planets around lower mass stars.
\end{abstract}

Subject headings: planetary systems: formation — stars: individual (HD 192699, HD 210702, HD 175541) — techniques: radial velocities

\section{INTRODUCTION}

Very little is known about the occurrence rate and orbital properties of planets around A-type stars, corresponding to stellar masses ranging from 1.6 to $3.0 M_{\odot}$. Inspection of the Catalog of Nearby Exoplanets (CNE) ${ }^{9}$ reveals that only six of the 173 stars with securely detected planetary companions have masses in excess of 1.6 $M_{\odot}$ (Butler et al. 2006). This small number of detections is not a true reflection of the occurrence of planets around A-type stars, but rather the result of a strong selection bias against early-type, main-sequence stars in precision Doppler surveys.

Measuring precise Doppler shifts of early-type dwarfs is complicated by their rotationally broadened spectral features, high surface temperatures, and high levels of excess radial velocity noise, or "jitter" (Saar et al. 1998; Wright 2005). Galland et al. (2005) find that Doppler precision for early-type dwarfs is limited to $\sim 40 \mathrm{~m} \mathrm{~s}^{-1}$ at spectral type F5 V, and $90-200 \mathrm{~m} \mathrm{~s}^{-1}$ for A stars, rendering Doppler measurements of these stars sensitive only to planets with large masses and short orbital periods. The lowest mass companion so far detected around an A star is the brown dwarf orbiting HD 180777 (Galland et al. 2006). Even though the 28 day orbital solution has a large velocity semi-

\footnotetext{
1 Based on observations obtained at the Lick Observatory, which is operated by the University of California, and W. M. Keck Observatory, which is operated jointly by the University of California and the California Institute of Technology.

2 Department of Astronomy, University of California, Berkeley, CA 94720.

3 Department of Physics and Astronomy, San Francisco State University, San Francisco, CA 94132.

4 Department of Earth and Planetary Sciences, Johns Hopkins University, Baltimore, MD 21218.

5 Department of Terrestrial Magnetism, Carnegie Institution of Washington, Washington, DC 20015-1305.

${ }^{6}$ Leiden Observatory, Leiden University, 2300 RA Leiden, Netherlands.

7 ZAH-Landessternwarte, 69117 Heidelberg, Germany.

${ }^{8} \mathrm{UCO} /$ Lick Observatory, University of California at Santa Cruz, Santa Cruz, CA 95064.

${ }^{9}$ For the updated catalog of extrasolar planet and their parameters see http:// exoplanets.org.
}

amplitude, $K=1200 \mathrm{~m} \mathrm{~s}^{-1}$, the signal is only a factor of 3 detection above the stellar jitter and measurement uncertainties.

Most of what is known about planet formation around intermediate-mass stars comes from two primary sources: direct imaging of disks around young stars and Doppler detections of planets around evolved stars. While A-type dwarfs are poor Doppler targets, their high intrinsic luminosities facilitate the detection and direct imaging of material in their circumstellar environments. More than a decade before the discoveries of the first extrasolar planets, evidence of planet formation outside of our solar system came from the infrared detection of collisiongenerated dust around the A-type, main-sequence stars Vega (Aumann et al. 1984) and $\beta$ Pic (Smith \& Terrile 1984). Since then, advances in high-contrast imaging have resulted in the detection of an optically thick disk around a pre-main-sequence Herbig Ae star (Perrin et al. 2006), as well as scattered light images of optically thin "debris disks" around 11 main-sequence stars - the majority of which have spectral types F5 V or earlier (Table 2 of Kalas et al. 2006; Schneider et al. 2006; Wahhaj et al. 2007 and references therein). Recent observations of the debris disk around the young A star Fomalhaut have revealed a perturbation in the disk structure that may be due to the influence of an orbiting Jovian planet (Kalas et al. 2005). Studying the relationships between the architectures of disks around young A stars and the distribution of planet properties around their older counterparts will provide key tests of planet formation models.

A key to finding planets around A stars using Doppler methods is provided by the effects of stellar evolution. As stars evolve away from the main sequence, they become cooler and rotate slower, which increases the number of narrow absorption lines in their spectra (Gray \& Nagar 1985; Schrijver \& Pols 1993; do Nascimento et al. 2000). Several Doppler surveys have focused on evolved, intermediate-mass stars on the red giant branch (Frink et al. 2002; D. S. Mitchell 2004, private communication; Hatzes et al. 2005; Lovis et al. 2005) and clump giant branch (Sato et al. 2003; Setiawan et al. 2003). These surveys have resulted in the discovery of six substellar companions orbiting former A-type 
TABLE 1

Planet Host Stars with $M_{*} \geq 1.6 M_{\odot}$

\begin{tabular}{cccccc}
\hline \hline HD & Spectral Type & $\begin{array}{c}M_{*} \\
\left(M_{\odot}\right)\end{array}$ & $\begin{array}{c}R_{*} \\
\left(R_{\odot}\right)\end{array}$ & $\begin{array}{c}a \\
(\mathrm{AU})\end{array}$ & References \\
\hline $13189 \ldots \ldots \ldots \ldots$. & K2 II & $2-6^{\mathrm{a}}$ & $\ldots$ & $1.5-2.2$ & 1 \\
$28305^{\mathrm{b}} \ldots \ldots \ldots \ldots$. & K0 III & 2.7 & 13.7 & 1.93 & 2 \\
$11977 \ldots \ldots \ldots \ldots .$. & G5 III & 1.9 & 13 & 1.93 & 3 \\
$62509^{\mathrm{c}} \ldots \ldots \ldots \ldots$. & K0 III & 1.86 & 8.8 & 1.7 & 4,5 \\
$210702 \ldots \ldots \ldots \ldots$. & K1 IV & 1.85 & 4.45 & 1.17 & 6 \\
$192699 \ldots \ldots \ldots \ldots$. & G8 IV & 1.68 & 3.90 & 1.16 & 6 \\
$175541 \ldots \ldots \ldots \ldots$. & G8 IV & 1.65 & 3.80 & 1.03 & 6 \\
$89744 \ldots \ldots \ldots \ldots .$. & F7 IV & 1.65 & 1.1 & 0.93 & 7 \\
$104985 \ldots \ldots \ldots \ldots$. & G9 III & 1.6 & 8.9 & 0.78 & 8 \\
\hline
\end{tabular}

Notes.-Excluded from this list of evolved, intermediate-mass planet host stars are $\gamma$ Cep A and HD 47536. The $\gamma$ Cep A discovery paper by Hatzes et al. (2003) cites a stellar mass of $1.59 M_{\odot}$. However, a recent dynamical analysis by Neuhäuser et al. (2007) yields a stellar mass of $1.40 \pm 0.12 M_{\odot}$. Setiawan et al. (2003) list a highly uncertain mass for the clump giant HD 47536, with a lower limit of $1.1 M_{\odot}$, which falls well below the $1.6 M_{\odot}$ cutoff used for this table.

${ }^{a}$ Schuler et al. (2005).

${ }^{\mathrm{b}} \mathrm{HD} 28305=\epsilon$ Tau.

${ }^{c}$ HD $62509=\beta \mathrm{Gem}=$ Pollux.

REFERENCES. - (1) Hatzes et al. 2005; (2) Sato et al. 2007; (3) Setiawan et al. 2005; (4) Hatzes et al. 2006; (5) Reffert et al. 2006; (6) this work; (7) Korzennik et al. 2000; (8) Sato et al. 2003.

stars (Table 1). That none of these planets would have been detectable during their host stars' main-sequence phases highlights the important role evolved stars play in the study of planets around intermediate-mass stars.

Here we present three new planet candidates around stars with $M_{*}>1.6 M_{\odot}$. These detections come from our precision Doppler survey of evolved stars on the subgiant branch of the H-R diagram. We discussed the selection criteria of our target stars in Johnson et al. (2006a) along with the discovery of an eccentric hot Jupiter orbiting the $1.28 M_{\odot}$ subgiant HD 185269 . We discuss our spectroscopic observations and Doppler measurement technique in $\S 2$. In $\S 3$ we present the characteristics of the host stars along with the orbital solutions for their planet candidates. We conclude with a comparison of the semimajor axis distributions of planets around A-type stars and lower mass stars in $\S 4$.

\section{OBSERVATIONS}

We are monitoring a sample of 159 evolved stars at Lick and Keck Observatories (Johnson et al. 2006a). At Lick Observatory, the Shane $3 \mathrm{~m}$ and $0.6 \mathrm{~m}$ Coude Auxiliary Telescopes (CAT) feed the Hamilton spectrometer (Vogt 1987), which has a resolution of $R \approx 50,000$ at $\lambda=5500 \AA$. Spectroscopic observations at Keck Observatory were obtained using the HIRES spectrometer with a resolution of $R \approx 80,000$ at $\lambda=5500 \AA$ (Vogt et al. 1994). Doppler shifts are measured from each spectrum using the iodine cell method described by Butler et al. (1996; see also Marcy \& Butler 1992). A temperature-controlled Pyrex cell containing gaseous iodine is placed at the entrance slit of the spectrometer. The dense set of narrow molecular lines imprinted on each stellar spectrum from 5000 to $6000 \AA$ provides a robust wavelength scale for each observation, as well as information about the shape of the spectrometer's instrumental response.

Traditionally, the Doppler shift of each stellar observation is made with respect to an observed, iodine-free stellar template spectrum. These template observations require higher signal and resolution than normal radial velocity observations, which leads to increased exposure times. Given our large target list and the small aperture of the CAT, obtaining an observed template for

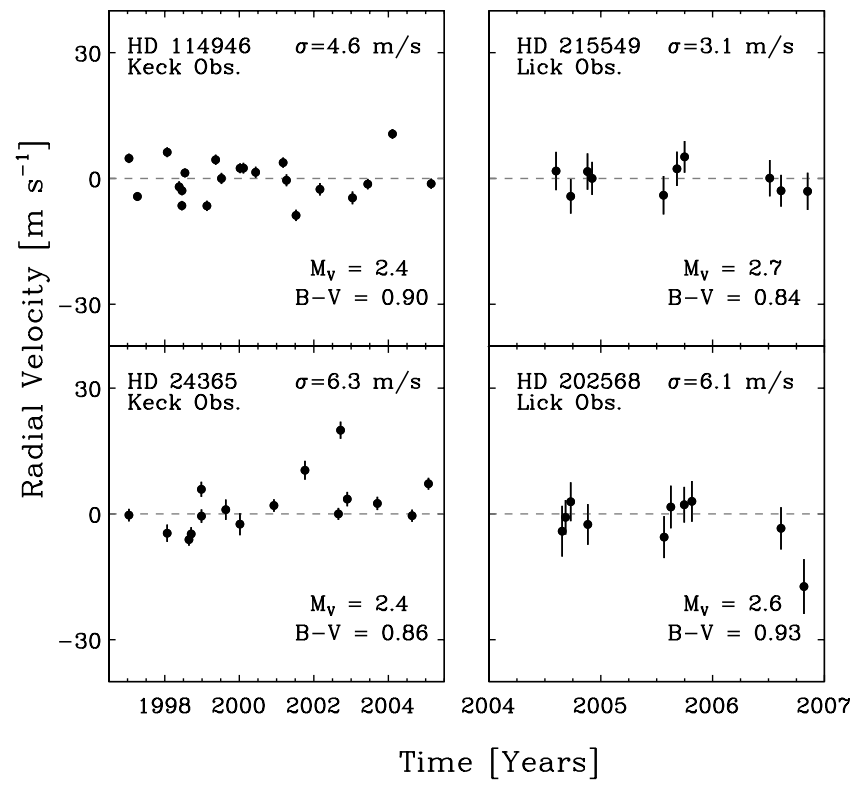

FIG. 1.-Radial velocity time series for four stable subgiants with $B-V$ colors and absolute visual magnitudes similar to those of the three subgiant planet host stars; $\sigma$ represents the standard deviation of the velocities about the mean (dashed lines). Observations from Keck (left panels) and Lick (right panels) show that subgiants in this region of the H-R diagram are typically stable to well within $10 \mathrm{~m} \mathrm{~s}^{-1}$ over timescales of many years.

each star would represent a prohibitive cost in observing time. We therefore perform a preliminary analysis of each star's observations using a synthetic, "morphed" template spectrum following the method described by Johnson et al. (2006b). Stars showing conspicuous Doppler variations are reanalyzed using a traditional, observed template to verify the signal and search for a full orbital solution.

Doppler measurements from Keck and Lick Observatories for four stable subgiants are shown in Figure 1. The error bars represent the internal uncertainties of each measurement, which are approximated by the weighted standard deviation of the mean velocity measured from each of the 700 individual $2 \AA$ wide chunks in each spectrum (Butler et al. 1996). We typically achieve internal measurement uncertainties of 1-2 $\mathrm{m} \mathrm{s}^{-1}$ for Keck observations and $3-5 \mathrm{~m} \mathrm{~s}^{-1}$ at Lick. Subgiants have an additional $4-6 \mathrm{~m} \mathrm{~s}^{-1}$ of "jitter"- -velocity scatter in excess of internal errors due to astrophysical sources such as pulsation and rotational modulation of surface features (Saar et al. 1998; Wright 2005). We therefore adopt a jitter value of $5 \mathrm{~m} \mathrm{~s}^{-1}$ for our subgiants, which is added in quadrature to the internal uncertainties of the measurements before searching for a best-fit orbital solution.

After determining the best-fit Keplerian solution using a Levenberg-Marquardt, least-squares minimization, we estimate the orbital parameter uncertainties using a bootstrap Monte Carlo method. We first subtract the best-fit Keplerian from the measured velocities. The residuals are then scrambled and added back to the original measurements, and a new set of orbital parameters is obtained. This process is repeated for 1000 trials, and the standard deviations of the parameters from all trials are adopted as the formal, $1 \sigma$ uncertainties.

\section{STELLAR PROPERTIES AND ORBIT SOLUTIONS}

\subsection{Estimates of Stellar Properties}

We estimated the stellar properties of our target stars using two primary methods: the LTE spectrum synthesis method (SME) 


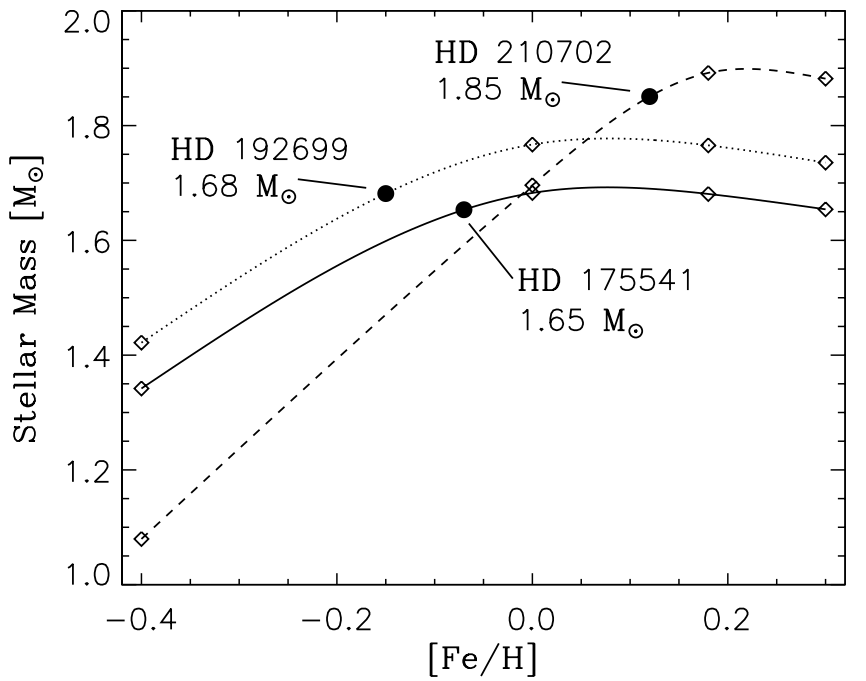

FIG. 2.-Illustration of the interpolation method employed to determine accurate stellar masses for the planet host stars. We estimated each star's mass, radius, and age by interpolating its Hipparcos $B-V$ color and absolute visual magnitude $M_{V}$ onto grids of four different metallicities: $[\mathrm{Fe} / \mathrm{H}]=-0.4,0.0,+0.18$, and +0.3 (open diamonds). For each star's measured value of $B-V$ and $M_{V}$, the three lines show the dependence of stellar mass on the measured $[\mathrm{Fe} / \mathrm{H}]$, estimated using a cubic spline interpolation between the diamonds. Similar dependencies were determined for stellar radii, luminosities, and ages.

described by Valenti \& Fischer (2005) and the Padova ${ }^{10}$ stellar interior models. The spectrum synthesis method uses a nonlinear least-squares algorithm to vary the parameters of a synthetic spectrum to search for a fit to an iodine-free stellar template spectrum. The free parameters in the fit are the abundances of heavy elements; effective surface temperature, $T_{\text {eff }}$; surface gravity, $\log g$; and broadening effects due to the star's projected rotation velocity, $V_{\text {rot }} \sin i$. Valenti \& Fischer (2005) estimate a precision of 0.04 dex in metallicity, $44 \mathrm{~K}$ in effective temperature, $0.3 \mathrm{dex}$ in $\log g$, and $0.5 \mathrm{~km} \mathrm{~s}^{-1}$ in rotational velocity. We used the SME-derived $T_{\text {eff }}$, Hipparcos absolutes magnitudes, and Flower (1996) bolometric corrections to calculate stellar luminosities and radii-and corresponding uncertainties - using the relationships derived by Valenti \& Fischer (2005).

To estimate stellar masses and ages we used the Padova theoretical stellar models, which have been transformed into several photometric systems by Girardi et al. (2002). Stellar properties can be inferred by interpolating a star's color, absolute magnitude, and metallicity onto these model grids. However, the Girardi et al. (2002) model grids are defined at widely spaced metallicity intervals, with $[\mathrm{Fe} / \mathrm{H}]=-0.4,0.0,+0.18$, and +0.30 . Since the uncertainties in our spectroscopically derived metallicity estimates are much less than the model grid intervals, and because the derived stellar properties do not vary linearly with $[\mathrm{Fe} / \mathrm{H}]$, we could not simply perform a linear, three-dimensional interpolation of $M_{V}, B-V$, and [Fe/H]. Instead, we first linearly interpolate the stars' colors and absolute magnitudes onto each of the four metallicity grids. We then use a cubic spline interpolation between the grid points to measure the desired stellar property (e.g., mass) at the star's measured $[\mathrm{Fe} / \mathrm{H}]$. Our procedure is illustrated in Figure 2, which shows stellar mass as a function of $[\mathrm{Fe} / \mathrm{H}]$ for each star's absolute magnitude and color. The same procedure was used for stellar ages.

We compared our interpolated stellar properties to the Takeda et al. (2007) theoretical interior models of the stars in the Spec-
TABLE 2

Stellar Parameters

\begin{tabular}{|c|c|c|c|}
\hline Parameter & HD 192699 & HD 210702 & HD 175541 \\
\hline$V \ldots$ & 6.44 & 5.93 & 8.03 \\
\hline$M_{V} \ldots \ldots \ldots \ldots \ldots \ldots \ldots \ldots \ldots$ & 2.30 & 2.19 & 2.49 \\
\hline$B-V$ & 0.867 & 0.951 & 0.869 \\
\hline Distance $(\mathrm{pc})$ & 67 & 56 & 128 \\
\hline$[\mathrm{Fe} / \mathrm{H}]]$ & $-0.15(0.04)$ & $+0.12(0.04)$ & $-0.07(0.04)$ \\
\hline$T_{\text {eff }}(\mathrm{K})$ & $5220(44)$ & $5010(44)$ & $5060(44)$ \\
\hline$V_{\mathrm{rot}} \sin i\left(\mathrm{~km} \mathrm{~s}^{-1}\right) \ldots \ldots \ldots$ & $1.9(0.5)$ & $1.7(0.5)$ & $2.9(0.5)$ \\
\hline $\log g$ & $3.44(0.3)$ & $3.29(0.3)$ & $3.52(0.3)$ \\
\hline$M_{*}\left(M_{\odot}\right)$ & $1.68(0.12)$ & $1.85(0.13)$ & $1.65(0.12)$ \\
\hline$R_{*}\left(R_{\odot}\right)$ & $3.90(0.06)$ & $4.45(0.07)$ & $3.80(0.09)$ \\
\hline$L_{*}\left(L_{\odot}\right)$ & $10.2(0.1)$ & $11.26(0.08)$ & $8.6(0.3)$ \\
\hline Age (Gyr) ....................... & $1.8(1.0)$ & $1.4(1.0)$ & $1.9(1.0)$ \\
\hline$S_{\mathrm{HK}}$ & 0.12 & 0.11 & 0.11 \\
\hline $\log R_{\mathrm{HK}}^{\prime}$ & -5.29 & -5.35 & -5.28 \\
\hline
\end{tabular}

troscopic Properties of Cool Stars (SPOCS) Catalog (Valenti \& Fischer 2005). We found a subset of 11 evolved stars in the catalog with $2.0<M_{V}<3.0$ and $0.7<B-V<1.1$. Differences between our inferred masses and those from Takeda et al. (2007) had an rms scatter of 7\%, with a median offset of $-2 \%$. Ages of this subset of evolved stars estimated by the two methods have a difference of -0.4 Gyr with an rms scatter of 1.1 Gyr. We therefore adopt fractional uncertainties of $7 \%$ for our derived masses and 1 Gyr for ages. We list the full set of derived stellar properties of the three candidate planet host stars in Table 2. We summarize each star's properties and orbital solution in the following subsections.

\subsection{HD 192699}

HD 192699 (HIP 99894) is listed with a G5 spectral type in the Hipparcos catalog, with $V=6.44, B-V=0.867$ and a parallax-based distance of 67 pc. ${ }^{11}$ However, no luminosity class is given. Based on its distance, we calculate $M_{V}=2.30$, which at its $B-V$ color places the star 3.7 mag above the mean mainsequence of stars in the solar neighborhood, as defined by Wright (2004). Based on its color and absolute magnitude, we find that HD 192699 is likely a G8 IV subgiant near the base of the red giant branch. Commensurate with its evolved status, HD 192699 is chromospherically inactive, with $S=0.12$ and $R_{\mathrm{HK}}^{\prime}=-5.29$ as measured from the $\mathrm{Ca}$ II $\mathrm{H}$ and $\mathrm{K}$ line core and averaged over all observations (Wright et al. 2004).

Based on our LTE spectral analysis, we find that HD 192699 is metal-poor, with $[\mathrm{Fe} / \mathrm{H}]=-0.15$, and slowly rotating, with $V_{\text {rot }} \sin i=1.9 \mathrm{~km} \mathrm{~s}^{-1}$. We interpolated the star's color, absolute magnitude, and metallicity onto the Girardi et al. (2002) theoretical stellar model grids using the method described in $\S 3.1$. Our interpolation yields a stellar mass $M_{*}=1.68 M_{\odot}$ and an age of 1.8 Gyr.

We began observing HD 192699 in 2004 May at Lick Observatory using the $3 \mathrm{~m}$ Shane Telescope and $0.6 \mathrm{~m}$ CAT. Table 3 lists our 34 velocity measurements, along with their times of observation and internal measurement uncertainties (without jitter). Our first seven observations, initially analyzed using a synthetic stellar template spectrum (Johnson et al. 2006b), showed correlated variations spanning two observing seasons. We obtained a high-quality observed template using the Shane $3 \mathrm{~m}$ telescope and initiated intensive follow-up observations during the 
TABLE 3

Radial Velocities for HD 192699

\begin{tabular}{|c|c|c|}
\hline $\mathrm{JD}-2,440,000$ & $\begin{array}{c}\mathrm{RV} \\
\left(\mathrm{m} \mathrm{s}^{-1}\right)\end{array}$ & $\begin{array}{l}\text { Uncertainty } \\
\left(\mathrm{m} \mathrm{s}^{-1}\right)\end{array}$ \\
\hline $13,155.988 \ldots \ldots$ & -60.66 & 5.14 \\
\hline $13,257.741 \ldots \ldots \ldots \ldots \ldots$ & 3.44 & 6.31 \\
\hline $13,522.921 \ldots \ldots \ldots \ldots \ldots$ & -47.84 & 4.90 \\
\hline $13,576.901 \ldots \ldots \ldots \ldots \ldots$ & -20.91 & 5.33 \\
\hline $13,619.774 \ldots \ldots \ldots \ldots \ldots$ & 13.49 & 4.90 \\
\hline $13,640.713 \ldots \ldots \ldots \ldots \ldots \ldots$ & 26.10 & 4.99 \\
\hline $13,668.641 \ldots \ldots \ldots \ldots \ldots \ldots$ & 19.02 & 4.66 \\
\hline $13,879.905 \ldots \ldots \ldots \ldots \ldots \ldots$ & -46.41 & 4.39 \\
\hline $13,891.854 \ldots \ldots \ldots \ldots \ldots \ldots$ & -34.19 & 5.46 \\
\hline $13,894.944 \ldots \ldots \ldots \ldots \ldots \ldots$ & -42.03 & 4.94 \\
\hline $13,895.904 \ldots \ldots \ldots \ldots \ldots$ & -56.83 & 4.71 \\
\hline $13,896.937 \ldots \ldots \ldots \ldots \ldots$ & -50.29 & 4.57 \\
\hline $13,905.962 \ldots \ldots \ldots \ldots \ldots$ & -28.97 & 6.96 \\
\hline 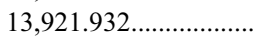 & -18.30 & 5.37 \\
\hline $13,922.851 \ldots \ldots \ldots \ldots \ldots \ldots$ & -1.89 & 4.63 \\
\hline $13,926.856 \ldots \ldots \ldots \ldots \ldots$ & -18.51 & 4.47 \\
\hline $13,951.801 \ldots \ldots \ldots \ldots \ldots$ & 11.70 & 4.62 \\
\hline $13,959.771 \ldots \ldots \ldots \ldots \ldots$ & -4.84 & 5.15 \\
\hline $13,966.784 \ldots \ldots \ldots \ldots \ldots$ & 8.99 & 5.63 \\
\hline $13,975.738 \ldots \ldots \ldots \ldots \ldots$ & 5.99 & 4.65 \\
\hline 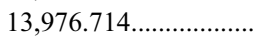 & 14.68 & 4.87 \\
\hline $13,998.725 \ldots \ldots \ldots \ldots \ldots$ & 25.11 & 5.28 \\
\hline $14,001.749 \ldots \ldots \ldots \ldots \ldots$ & 34.99 & 4.68 \\
\hline $14,020.654 \ldots \ldots \ldots \ldots \ldots$ & 49.36 & 5.02 \\
\hline $14,021.682 \ldots \ldots \ldots \ldots \ldots$ & 25.41 & 5.42 \\
\hline $14,034.638 \ldots \ldots \ldots \ldots \ldots$ & 18.33 & 5.23 \\
\hline $14,035.671 \ldots \ldots \ldots \ldots \ldots \ldots$ & 9.67 & 5.46 \\
\hline $14,039.603 \ldots \ldots \ldots \ldots \ldots$ & -2.60 & 5.69 \\
\hline $14,046.658 \ldots \ldots \ldots \ldots \ldots \ldots$ & 6.44 & 5.05 \\
\hline $14,049.615 \ldots \ldots \ldots \ldots \ldots \ldots$ & 5.84 & 5.60 \\
\hline $14,059.610 \ldots \ldots \ldots \ldots \ldots \ldots$ & -5.29 & 4.55 \\
\hline $14,070.664 \ldots \ldots \ldots \ldots \ldots \ldots$ & -16.58 & 5.30 \\
\hline $14,072.612 \ldots \ldots \ldots \ldots \ldots$ & -18.70 & 5.17 \\
\hline $14,170.058 \ldots \ldots \ldots \ldots \ldots$ & -78.23 & 5.92 \\
\hline
\end{tabular}

fall of 2006 observing season. The Keplerian signal is visible to the eye (Fig. 3), obviating a periodogram analysis.

The best-fit Keplerian orbit has a period of $P=351.5$ days, velocity amplitude $K=51.3 \mathrm{~m} \mathrm{~s}^{-1}$, and eccentricity $e=0.149 \pm$ 0.06 . With an assumed stellar mass of $1.68 M_{\odot}$, we estimate a minimum planet mass $M_{P} \sin i=2.5 M_{\mathrm{J}}$ and orbital separation $a=1.16 \mathrm{AU}$. The fit has rms $=9.2 \mathrm{~m} \mathrm{~s}^{-1}$ and a reduced $\sqrt{\chi_{\nu}^{2}}=$ 1.14 , consistent with the measurement errors and jitter. The full set of orbital parameters and uncertainties is listed in Table 4 .

\subsection{HD 210702}

HD 210702 (HIP 109577, HR 8461) is listed in the Hipparcos catalog as a $\mathrm{K} 1$ star (no luminosity class given) with $V=5.93$, $B-V=0.951$, and a parallax-based distance of $56 \mathrm{pc}$. Given its distance and apparent magnitude, we calculate an absolute magnitude $M_{V}=2.19$, which places it $4.2 \mathrm{mag}$ above the average main sequence of stars in the solar neighborhood (Wright 2004). We therefore estimate that HD 210702 is a class K1 IV subgiant near the base of the red giant branch.

Based on our LTE spectral analysis, we find that HD 210702 is somewhat metal-rich, with $[\mathrm{Fe} / \mathrm{H}]=+0.12 \pm 0.04$, and slowly rotating, with $V_{\mathrm{rot}} \sin i=1.7 \mathrm{~km} \mathrm{~s}^{-1}$. Our interpolation of the star's color, absolute magnitude, and metallicity onto the Girardi et al. (2002) stellar model grids yields a stellar mass $M_{*}=1.85 M_{\odot}$ and

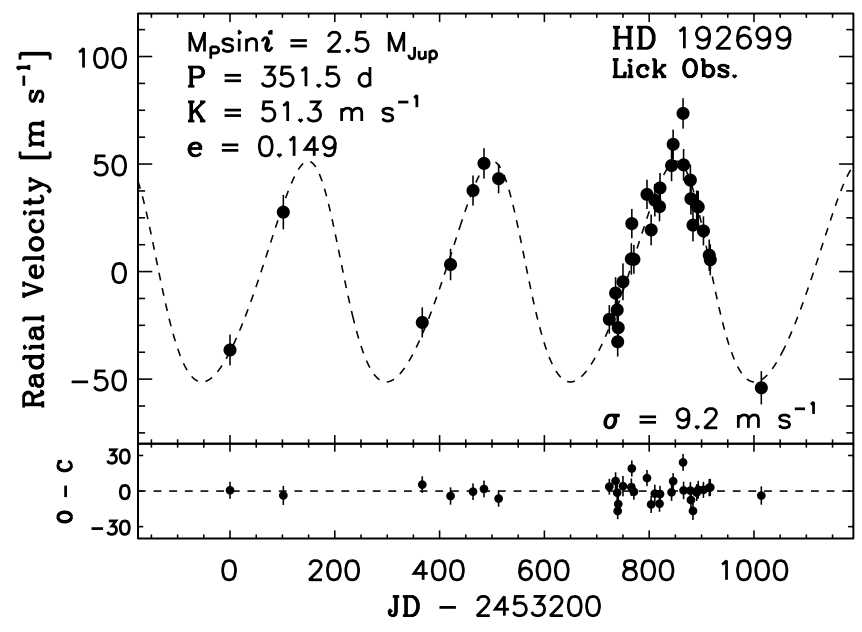

FIG. 3.- Radial velocity time series for HD 192699 measured at Lick Observatory. The error bars reflect the quadrature sum of the internal measurement uncertainties and $5 \mathrm{~m} \mathrm{~s}^{-1}$ of jitter. The dashed line shows the best-fit orbital solution, which has $\sqrt{\chi_{\nu}^{2}}=1.14$.

an age of 1.4 Gyr. Consistent with its post-main-sequence evolutionary status, HD 210702 is chromospherically inactive with $S=0.11$ and $R_{\mathrm{HK}}^{\prime}=-5.35$, as measured from its $\mathrm{Ca}$ II $\mathrm{H}$ and $\mathrm{K}$ emission (Wright et al. 2004). The other stellar parameters derived from our spectral analysis and stellar model interpolation are listed in Table 2.

We began monitoring HD 210702 in 2004 August at Lick Observatory. The first nine observations were Doppler-analyzed using a synthetic template, and showed excessive variability with $\mathrm{rms}=19 \mathrm{~m} \mathrm{~s}^{-1}$. We then obtained a traditional, observed template to confirm the variations with higher Doppler precision. The full set of velocities is listed in Table 5 (without jitter) and plotted in Figure 4. The error bars in Figure 4 have been augmented by adding $5 \mathrm{~m} \mathrm{~s}^{-1}$ of jitter in quadrature to the internal measurement uncertainties.

The best-fit Keplerian orbital solution is shown in Figure 4 overplotted on the velocities. The solution has a 341.1 day period, an eccentricity $e=0.152 \pm 0.08$, and a semiamplitude $K=39.2 \mathrm{~m} \mathrm{~s}^{-1}$. The fit residuals have rms $=7.4 \mathrm{~m} \mathrm{~s}^{-1}$ and reduced $\sqrt{\chi_{\nu}^{2}}=1.07$, consistent with the internal measurement uncertainties and jitter. Assuming a stellar mass $M_{*}=1.85 M_{\odot}$, the best-fit solution yields a relative separation $a=1.17 \mathrm{AU}$.

We find the inclusion of a linear trend in the orbital solution yields a slight improvement in the quality of fit, decreasing the

TABLE 4

Orbital Parameters

\begin{tabular}{|c|c|c|c|}
\hline Parameter & HD 192699b & HD 210702b & HD $175541 b$ \\
\hline$P$ (day) & $351.5(6)$ & $341.1(7)$ & $297.3(6)$ \\
\hline$T_{p}^{\mathrm{a}}(\mathrm{JD}) \ldots \ldots \ldots \ldots \ldots$ & $2,452,994(30)$ & $2,453,118(40)$ & $2,450,213(20)$ \\
\hline e & $0.149(0.06)$ & $0.152(0.08)$ & $0.33(0.2)$ \\
\hline$K_{1}\left(\mathrm{~m} \mathrm{~s}^{-1}\right) \ldots \ldots \ldots \ldots \ldots$ & $51.3(5)$ & $39.2(4)$ & $14.0(2)$ \\
\hline$\omega(\operatorname{deg})$ & $54(30)$ & $301(30)$ & $183(30)$ \\
\hline$M_{P} \sin i\left(M_{\mathrm{J}}\right) \ldots \ldots \ldots \ldots$ & 2.5 & 2.0 & 0.61 \\
\hline$a(\mathrm{AU}) \ldots \ldots$ & 1.16 & 1.17 & 1.03 \\
\hline Fit rms $\left(\mathrm{m} \mathrm{s}^{-1}\right) \ldots \ldots \ldots$ & 9.2 & 7.4 & 5.6 \\
\hline$\sqrt{\chi_{\nu}^{2}} \ldots \ldots \ldots \ldots \ldots \ldots \ldots \ldots$ & 1.14 & 1.07 & 1.01 \\
\hline$N_{\text {obs }} \ldots \ldots$ & 34 & 29 & 29 \\
\hline
\end{tabular}

a Time of periastron passage. 
TABLE 5

Radial Velocities for HD 210702

\begin{tabular}{|c|c|c|}
\hline $\mathrm{JD}-2,440,000$ & $\begin{array}{c}\mathrm{RV} \\
\left(\mathrm{m} \mathrm{s}^{-1}\right)\end{array}$ & $\begin{array}{l}\text { Uncertainty } \\
\qquad\left(\mathrm{m} \mathrm{s}^{-1}\right)\end{array}$ \\
\hline $13,241.863 \ldots .$. & 18.13 & 3.32 \\
\hline $13,256.776 \ldots \ldots \ldots \ldots \ldots$ & 9.30 & 3.91 \\
\hline $13,310.752 \ldots \ldots \ldots \ldots \ldots$ & -16.07 & 4.33 \\
\hline $13,342.598 \ldots \ldots \ldots \ldots \ldots$ & -0.18 & 3.37 \\
\hline $13,569.930 \ldots \ldots \ldots \ldots \ldots \ldots$ & 42.99 & 4.77 \\
\hline $13,573.904 \ldots \ldots \ldots \ldots \ldots \ldots$ & 29.79 & 5.13 \\
\hline $13,574.927 \ldots \ldots \ldots \ldots \ldots$ & 38.71 & 4.47 \\
\hline $13,575.897 \ldots \ldots \ldots \ldots \ldots$ & 29.80 & 4.41 \\
\hline $13,640.779 \ldots \ldots \ldots \ldots \ldots$ & 10.99 & 5.45 \\
\hline $13,922.930 \ldots \ldots \ldots \ldots \ldots \ldots$ & 28.01 & 3.39 \\
\hline $13,959.860 \ldots \ldots \ldots \ldots \ldots . . .$. & 0.18 & 3.22 \\
\hline $13,975.798 \ldots \ldots \ldots \ldots \ldots \ldots$ & -1.47 & 3.54 \\
\hline $13,976.764 \ldots \ldots \ldots \ldots \ldots \ldots$ & -8.51 & 3.20 \\
\hline $13,998.768 \ldots \ldots \ldots \ldots \ldots$ & -10.73 & 3.52 \\
\hline $14,001.789 \ldots \ldots \ldots \ldots \ldots$ & -13.09 & 3.61 \\
\hline $14,020.721 \ldots \ldots \ldots \ldots \ldots$ & -15.97 & 3.73 \\
\hline $14,021.735 \ldots \ldots \ldots \ldots \ldots$ & -11.53 & 4.38 \\
\hline $14,034.695 \ldots \ldots \ldots \ldots \ldots \ldots$ & -22.55 & 4.41 \\
\hline $14,046.694 \ldots \ldots \ldots \ldots \ldots . .$. & -31.56 & 3.40 \\
\hline $14,059.673 \ldots \ldots \ldots \ldots \ldots$ & -14.62 & 3.57 \\
\hline $14,070.705 \ldots \ldots \ldots \ldots \ldots$ & -11.10 & 3.70 \\
\hline $14,092.594 \ldots \ldots \ldots \ldots \ldots$ & 1.07 & 4.06 \\
\hline $14,097.649 \ldots \ldots \ldots \ldots \ldots . .$. & -2.40 & 3.31 \\
\hline $14,098.603 \ldots \ldots \ldots \ldots \ldots . .$. & 1.90 & 3.78 \\
\hline $14,103.598 \ldots \ldots \ldots \ldots \ldots \ldots$ & 9.35 & 3.65 \\
\hline $14,109.626 \ldots \ldots \ldots \ldots \ldots$ & 9.02 & 5.12 \\
\hline $14,117.595 \ldots \ldots \ldots \ldots \ldots \ldots$ & 22.46 & 5.58 \\
\hline $14,136.600 \ldots \ldots \ldots \ldots \ldots$ & 38.79 & 3.09 \\
\hline $14,197.033 \ldots \ldots \ldots \ldots \ldots$ & 53.95 & 4.26 \\
\hline
\end{tabular}

rms scatter of the residuals from $7.4 \mathrm{~m} \mathrm{~s}^{-1}$ to $6.7 \mathrm{~m} \mathrm{~s}^{-1}$, and the reduced $\sqrt{\chi_{\nu}^{2}}$ from 1.07 to 1.00 after accounting for the extra free parameter in the Keplerian-plus-trend model. We tested the validity of the trend using the prescription of Wright et al. (2007) and found a false-alarm probability (FAP) of $49 \%$. The large FAP indicates that the apparent linear trend is likely due to noise rather than an additional orbital companion. Indeed, the trend appears to be driven primarily by the three outliers near $\mathrm{JD}=100,400$, and 800 (Fig. 4). We therefore favor the singleplanet Keplerian model summarized in Table 4.



Fig. 4.-Radial velocity time series for HD 210702 measured at Lick Observatory. The dashed line shows the best-fit orbital solution, which has $\sqrt{\chi_{\nu}^{2}}=1.07$.
TABLE 6

RADIAL VeLocities FOR HD 175541

\begin{tabular}{|c|c|c|}
\hline $\mathrm{JD}-2,440,000$ & $\begin{array}{c}\mathrm{RV} \\
\left(\mathrm{m} \mathrm{s}^{-1}\right)\end{array}$ & $\begin{array}{l}\text { Uncertainty } \\
\left(\mathrm{m} \mathrm{s}^{-1}\right)\end{array}$ \\
\hline $10,283.952 \ldots \ldots \ldots \ldots \ldots \ldots$ & 5.14 & 1.46 \\
\hline $10,603.027 \ldots \ldots \ldots \ldots \ldots \ldots$ & 10.95 & 1.63 \\
\hline $10,665.908 \ldots \ldots \ldots \ldots \ldots \ldots$ & 12.81 & 1.63 \\
\hline $11,011.878 \ldots \ldots \ldots \ldots \ldots \ldots$ & 0.94 & 2.19 \\
\hline $11,069.830 \ldots \ldots \ldots \ldots \ldots$ & -14.86 & 2.17 \\
\hline $11,367.894 \ldots \ldots \ldots \ldots \ldots \ldots$ & -7.68 & 1.98 \\
\hline $11,410.857 \ldots \ldots \ldots \ldots \ldots \ldots$ & -12.21 & 2.29 \\
\hline $11,441.759 \ldots \ldots \ldots \ldots \ldots$ & -5.03 & 1.96 \\
\hline $11,705.979 \ldots \ldots \ldots \ldots \ldots \ldots$ & -15.48 & 2.25 \\
\hline $11,984.164 \ldots \ldots \ldots \ldots \ldots \ldots$ & -13.59 & 2.50 \\
\hline $12,004.126 \ldots \ldots \ldots \ldots \ldots \ldots$ & -15.69 & 2.18 \\
\hline $12,008.065 \ldots \ldots \ldots \ldots \ldots \ldots$ & -19.07 & 2.17 \\
\hline $12,009.118 \ldots \ldots \ldots \ldots \ldots \ldots$ & -28.52 & 2.44 \\
\hline $12,030.989 \ldots \ldots \ldots \ldots \ldots$ & -4.26 & 1.67 \\
\hline $12,095.962 \ldots \ldots \ldots \ldots \ldots \ldots$ & 5.95 & 1.94 \\
\hline $12,098.016 \ldots \ldots \ldots \ldots \ldots \ldots$ & -2.49 & 2.09 \\
\hline $12,099.039 \ldots \ldots \ldots \ldots \ldots$ & 10.75 & 1.71 \\
\hline $12,100.956 \ldots \ldots \ldots \ldots \ldots \ldots$ & 2.62 & 1.94 \\
\hline $12,127.874 \ldots \ldots \ldots \ldots \ldots \ldots$ & 5.87 & 2.05 \\
\hline $12,391.132 \ldots \ldots \ldots \ldots \ldots \ldots$ & 3.80 & 2.13 \\
\hline $12,488.898 \ldots \ldots \ldots \ldots \ldots \ldots$ & 10.77 & 1.78 \\
\hline $12,573.725 \ldots \ldots \ldots \ldots \ldots$ & -9.38 & 1.82 \\
\hline $12,833.948 \ldots \ldots \ldots \ldots \ldots \ldots$ & -1.19 & 1.82 \\
\hline $13,239.787 \ldots \ldots \ldots \ldots \ldots \ldots$ & 0.00 & 1.80 \\
\hline $13,546.914 \ldots \ldots \ldots \ldots \ldots \ldots$ & 7.36 & 1.98 \\
\hline $13,603.852 \ldots \ldots \ldots \ldots \ldots \ldots$ & 15.72 & 1.89 \\
\hline $13,807.150 \ldots \ldots \ldots \ldots \ldots \ldots$ & -3.79 & 1.93 \\
\hline $13,932.962 \ldots \ldots \ldots \ldots \ldots$ & 16.87 & 1.76 \\
\hline $13,968.920 \ldots \ldots \ldots \ldots \ldots$ & 12.16 & 1.83 \\
\hline
\end{tabular}

\section{4. $H D 175541$}

HD 175541 (HIP 92895) is listed in the Hipparcos catalog as a G8 V star with $V=8.03, B-V=0.869$ and a parallax-based distance of $128 \mathrm{pc}$ (ESA 1997). Given its distance, the star has $M_{V}=2.49$, placing it $3.5 \mathrm{mag}$ above the mean main-sequence of stars in the solar neighborhood (Wright 2004). Like most evolved stars, HD 175541 is chromospherically quiet with $S=0.11$ and $R_{\mathrm{HK}}^{\prime}=-5.28$ (Wright et al. 2004). Its low chromospheric activity and location in the H-R diagram indicate that HD 175541 is most likely a luminosity class IV star on the subgiant branch, rather than a class $\mathrm{V}$ dwarf.

HD 175541 is listed in the SPOCS Catalog (Valenti \& Fischer $2005)$ with a metal abundance slightly below solar $([\mathrm{Fe} / \mathrm{H}]=$ $-0.07 \pm 0.04)$ and projected rotational velocity $V_{\text {rot }} \sin i=$ $2.9 \mathrm{~km} \mathrm{~s}^{-1}$. Interpolation of the star's $B-V$ color, absolute magnitude and metallicity onto the Girardi et al. (2002) stellar model grids yields a stellar mass $M_{*}=1.65 M_{\odot}$ and an age of 1.9 Gyr. The interior models of Takeda et al. (2007) yield $M_{*}=1.52 M_{\odot}$. The SPOCS Catalog lists $M_{*}=1.74 M_{\odot}$ (Valenti $\&$ Fischer 2005). The variance of these different mass estimates is $0.1 M_{\odot}$, consistent with our estimate of the uncertainty in $\S 3.1$. The other stellar properties are listed in Table 2.

HD 175541 was one of the original stars added to the CCPS Keck program in 1996, and was subsequently added to our list of intermediate-mass stars in 2004. Table 6 lists our 29 Doppler measurements along with their observation dates and internal uncertainties (without jitter). Figure 5 shows that the rms scatter of the velocity measurements is a factor of 6 greater than the mean internal uncertainty $\left(\bar{\sigma}_{v} \approx 2 \mathrm{~m} \mathrm{~s}^{-1}\right)$, and 2-3 times larger than the rms scatter of stable Keck subgiants (Fig. 1). A Lombe-Scargle 


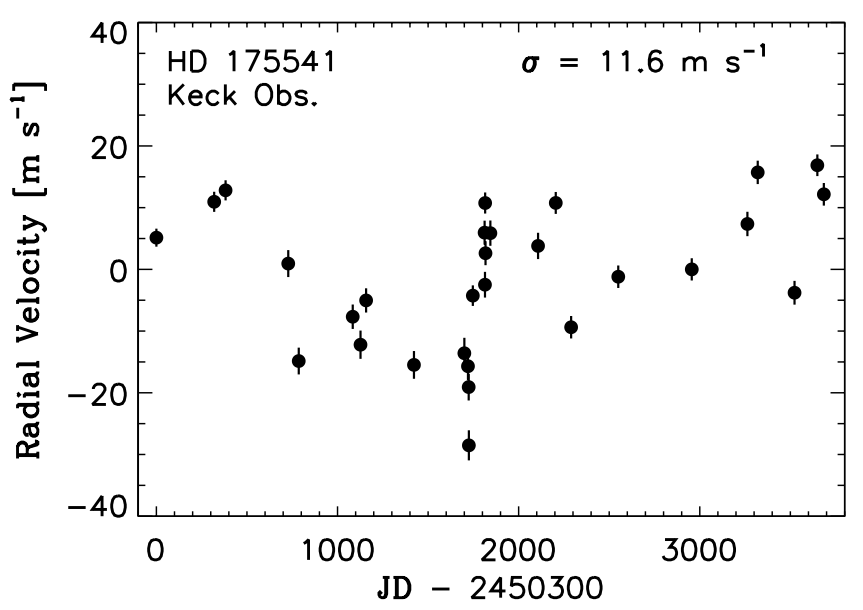

FIG. 5.-Radial velocities for HD 175541 measured at Keck Observatory. The error bars represent the internal uncertainty of each measurement without accounting for stellar jitter.

periodogram analysis of the velocities reveals a pronounced peak near $P=300$ days, with an FAP $<0.1 \%$ (Fig. 6).

To search for the best-fit orbital solution, we added $5 \mathrm{~m} \mathrm{~s}^{-1}$ of jitter in quadrature to the internal measurement uncertainties. We find that a Keplerian with $P=297.3$ days, $K=14.0 \mathrm{~m} \mathrm{~s}^{-1}$, and $e=0.33$ provides the best fit to the data, resulting in $\mathrm{rms}=$ $5.6 \mathrm{~m} \mathrm{~s}^{-1}$ and $\sqrt{\chi_{\nu}^{2}}=1.01$. Figure 7 shows the radial velocities phased at $P=297.3$ day, along with the best-fit orbital solution (the gray points show the measurements at phases outside of phases 0.0 and 1.0, in order to guide the eye). Assuming a stellar mass of $1.65 M_{\odot}$, we estimate a minimum planet mass $M_{P} \sin i=$ $0.61 M_{\mathrm{J}}$ and orbital separation $a=1.03 \mathrm{AU}$.

While the strong periodogram peak and low $\sqrt{\chi_{\nu}^{2}}$ are indicative of a correlated signal resulting from an orbiting planet, it is still possible that random variability could conspire to produce a false periodicity in our sparse series of measurements. To test the null hypothesis, we used the "scrambled velocities" false-


FIG. 6.-Top: Periodogram analysis of the RV time series of HD 175541. A strong peak is visible near $P=300$ days with an analytic FAP $<0.1 \%$. Bottom: Empirical assessment of the FAP of the best-fit Keplerian model. The original, unscrambled velocities yield an orbital solution with $\sqrt{\chi_{\nu}^{2}}=1.01$ (arrow). The histogram shows the distribution of $\sqrt{\chi_{\nu}^{2}}$ obtained from the best-fit orbital solution for each of the scrambled-velocities trials. None of the $10^{4}$ trials produced a value of $\sqrt{\chi_{\nu}^{2}}$ lower than the value obtained from the original time series, resulting in FAP $<0.01 \%$ (see $\S 3.4$ ).

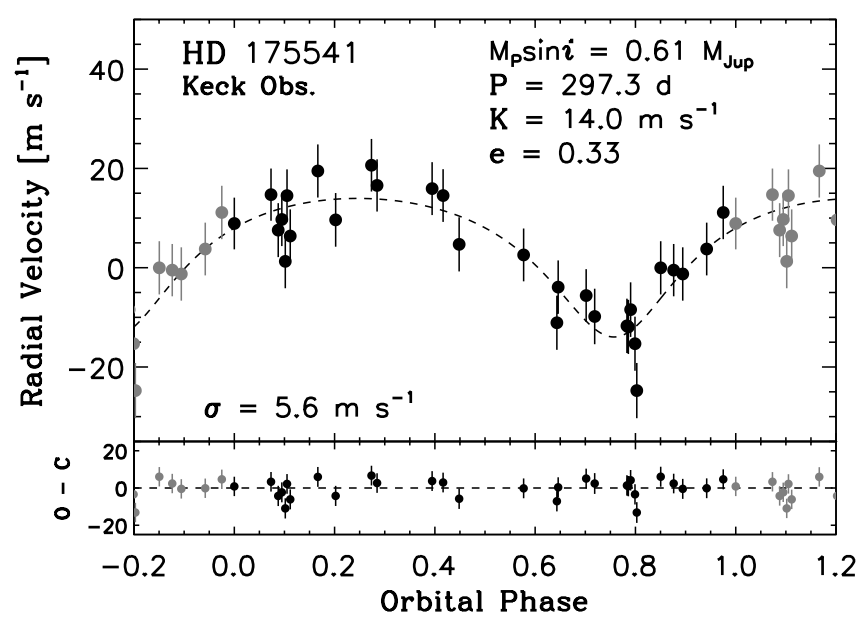

FIG. 7. - Radial velocity observations of HD 175541 phased at $P=297.3$ days. The gray points lie outside of phases 0.0 and 1.0 and are included to guide the eye. The dashed line shows the best-fit Keplerian orbital solution, which has $\sqrt{\chi_{\nu}^{2}}=1.01$.

alarm test described by Marcy et al. (2005). For $10^{4}$ separate trials, we held the observation times constant and scrambled the order of the measurements using a pseudo random number generator. This has the effect of keeping the sampling constant while removing any true temporal coherence, if such a signal exists. For each of the scrambled trials, we perform a full search for the best-fit Keplerian orbital solution-with jitter-and record the $\sqrt{\chi_{\nu}^{2}}$ from the fit.

The distribution of $\sqrt{\chi_{\nu}^{2}}$ generated from the scrambledvelocity trials is then compared to the fit obtained from the original time series, as shown in the lower panel of Figure 6 . None of the $10^{4}$ scrambled trials produced a $\sqrt{\chi_{\nu}^{2}}$ equal to or lower than the best-fit solution to the original time series, resulting in FAP $<0.01 \%$. From this test, we conclude that the temporally correlated signal seen in the velocity time series is likely real, rather than an artifact of random noise. We find that the best explanation of the periodic signal is the presence of an unseen planetary companion orbiting HD 175541.

\section{SUMMARY AND DISCUSSION}

We present precision Doppler measurements of three intermediate-mass subgiants that show periodic variations in their radial velocities consistent with planet-mass orbital companions. Interpolation of the stars' absolute magnitudes, colors and metallicities onto the Girardi et al. (2002) stellar interior models shows that all three stars have masses ranging from 1.65 to $1.85 M_{\odot}$. Figure 8 shows these massive host stars on an H-R diagram, along with their theoretical evolution tracks. Following the tracks back to the zero-age main sequence reveals that these present-day subgiants were originally early-type dwarfs with $B-V \lesssim 0.2$ and spectral types ranging from $\mathrm{A} 2 \mathrm{~V}$ to $\mathrm{A} 5 \mathrm{~V}$. The three long-period planets presented here would not have been detectable during their stars' main-sequence phases due to the jitter and rotational line broadening typical for intermediatemass dwarfs. These planets orbiting "retired" A stars illustrate how evolved stars provide a unique window into stellar mass and planetary domains otherwise inaccessible to Doppler-based planet searches.

There are now nine former A-type stars $\left(1.6 \lesssim M_{*}<3.0 M_{\odot}\right)$ with planetary companions. We list some of the properties of these massive host stars and their planets in Table 1. All nine planets orbit beyond $\sim 0.78$ AU from their stars. This paucity of planets with semimajor axes $a<0.78 \mathrm{AU}$ is unlikely due to a 


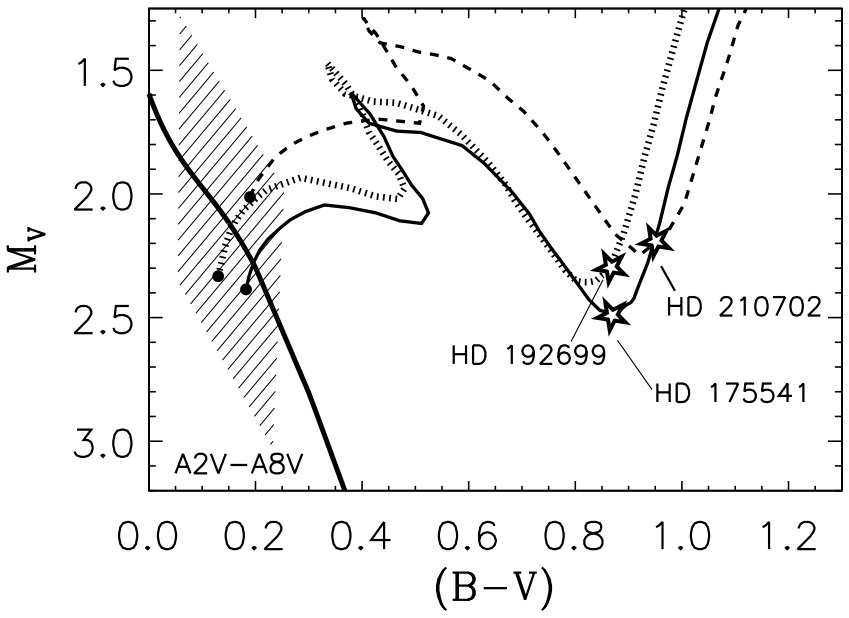

Fig. 8.-H-R diagram illustrating the properties of the three subgiant planet host stars ( pentagrams) compared to their main-sequence progenitors ( filled circles). The connecting lines represent each star's Girardi et al. (2002) theoretical mass track, interpolated for that star's metallicity. The thick, diagonal line is the theoretical zero-age main sequence for $[\mathrm{Fe} / \mathrm{H}]=0.0$. The hatched region shows the approximate range of colors and magnitudes of stars with spectral types A2 V-A8 V.

detection bias. For a given planet mass and stellar mass, the velocity semiamplitude of a star scales as $K \sim a^{-1 / 2}$, making planets in smaller orbits easier to detect. The detectability of close-in planets is also facilitated by the increased number of orbital cycles that are observable over a given time span.

We consider two possible explanations for the observed lack of close-in planets around intermediate-mass stars. The first possibility is that planets around A-type stars have the same semimajor axis distribution as planets orbiting lower mass stars, but the closein planets were destroyed by the expanding atmospheres of their giant host stars. Alternatively, planets orbiting A-type stars may have a different semimajor axis distribution than lower mass stars, with planets residing preferentially in long-period orbits beyond $\sim 0.8$ AU.

These possibilities can be explored by comparing the properties of planets in Table 1 to planets orbiting lower mass stars listed in the CNE. We exclude extremely low-mass planets with $K<15 \mathrm{~m} \mathrm{~s}^{-1}$ that would not be easily detectable around higher mass subgiants and giants. We use a one-sided KolmogorovSmirnov (K-S; Press et al. 1992) test to compare the semimajor axis distributions of planets around intermediate-mass and lower mass stars. We find the probability that the two distributions are identical is only $0.06 \%$. Under the assumption that the semimajor axis distribution of planets is independent of stellar mass, shortperiod planets orbiting evolved A-type stars must be efficiently destroyed by the expanding atmospheres of their giant host stars. The validity of this hypothesis depends on whether the radii of $\sim 2 M_{\odot}$ giants are large enough to engulf planets out to $0.8 \mathrm{AU}$.

Figure 9 shows the evolution of the radius of a $2.0 M_{\odot}$ star according to the Girardi et al. (2002) stellar evolution models. As the star crosses the Hertzsprung gap during its subgiant phase, its radius remains nearly constant at $a \approx 5 R_{\odot}=0.023 \mathrm{AU}$, which is within the orbit of a $P=3$ day hot Jupiter. Not until the star begins to ascend the RGB does its outer atmosphere begin to encroach on the orbits of short-period planets. But even at the tip of the RGB (near the helium flash), the radius of a $2 M_{\odot}$ star is only at the distance of a 10 day hot Jupiter at $a \approx 26 R_{\odot}=$ $0.12 \mathrm{AU}$ (the radius of a $2.5 M_{\odot}$ red giant is not much larger at

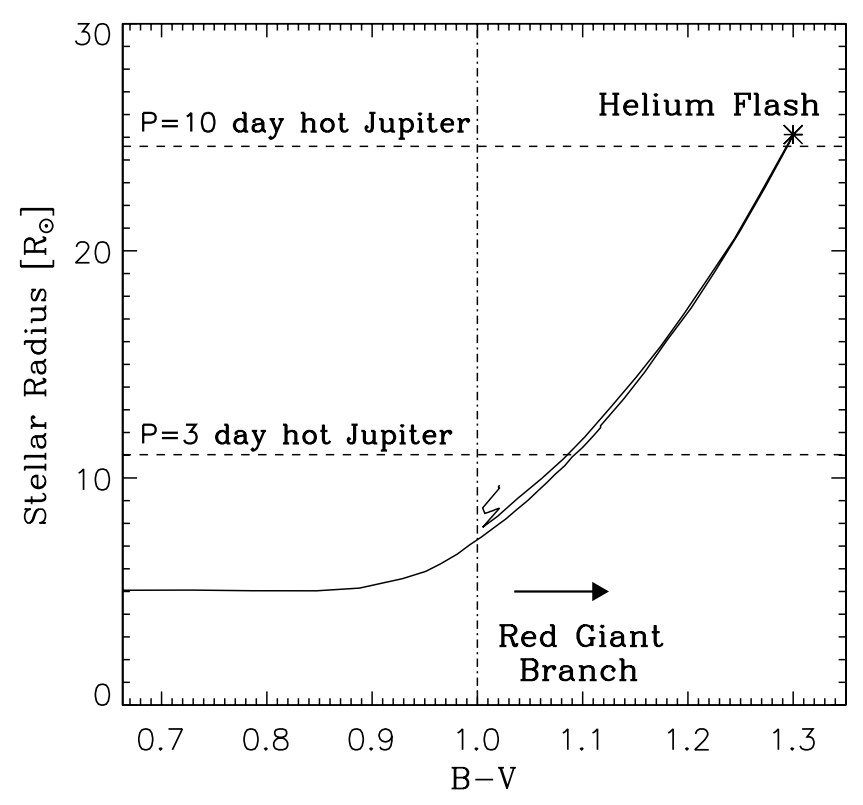

FIG. 9.- Radius of a $1.9 M_{\odot}$ star as it evolves off of the main sequence (becoming redder). The horizontal dashed line s depict the semimajor axes of planets with periods of 3 and 10 days. The vertical dot-dashed line shows the approximate $B-V$ color of the star as it begins to ascend the red giant branch. Because the radii of subgiants are small enough to avoid interference with closein planets, our Doppler survey is sensitive to the same range of orbital separations as surveys of main-sequence stars. Planets with $a \lesssim 30 R_{\odot}$ may be destroyed by the expansion of their host stars on the red giant branch.

$\left.a \approx 32 R_{\odot}=0.15 \mathrm{AU}\right)$. Thus, engulfment cannot be solely responsible for the lack of close-in planets around subgiants and $\mathrm{K}$ giants. Indeed, engulfment can only be important for four of the stars in Table 1: the post-helium-flash clump giants HD 104985, HD 11977, and $\epsilon$ Tau, and HD 13189, which has a poorly constrained radius due to its highly uncertain parallax.

The evolution of planetary orbits from $0.05-0.15 \mathrm{AU}$ in the presence of an expanding stellar atmosphere has not been examined in detail. The effects of planet engulfment on its host star have been studied by Siess \& Livio (1999), but a key assumption in their model is that the substellar companion is destroyed. Since it is unclear what happens to a planet when it interacts with the atmosphere of its expanding host star, we simply assume that planets orbiting within the radius of a giant star are destroyed. ${ }^{12}$ Under this assumption, we would expect a deficiency of hot Jupiters around clump giants out to $\sim 0.15 \mathrm{AU}$, but no corresponding deficiency around subgiants and $\mathrm{K}$ giants.

We now analyze the lack of close-in planets around the sample in Table 1 accounting the possible destruction of hot Jupiters around clump giants. For subgiants and giants we can use the K-S test as before, which yields a probability of $0.7 \%$ that the semimajor axis distribution is the same as lower mass stars in the CNE. For clump giants we exclude planets from the CNE with $a<$ $0.15 \mathrm{AU}$, and the corresponding probability from the K-S test is $1.7 \%$. Thus, the distribution of close-in planets around former A-type stars remains inconsistent with the distribution of planets in the CNE. Since engulfment does not provide an adequate explanation for the lack of close-in planets in Table 1, we are left with the possibility that the semimajor axis distribution of

12 Maxted et al. (2006) discovered a short-period substellar companion that apparently survived engulfment as its parent star evolved into a white dwarf. However, no Jovian planet has yet been detected around a white dwarf. 
planets around A-type stars is significantly different than the distribution around lower mass stars $\left(M_{*}<1.6 M_{\odot}\right)$.

Differences between the semimajor axes of planets around stars of various masses has previously been investigated by Burkert \& Ida (2007). From their study of the orbital properties of known exoplanets, they find evidence of a gap in the semimajor axis distribution around stars with masses $M_{*} \geq 1.2 M_{\odot}$, with fewer planets between 0.08 and $0.6 \mathrm{AU}$ compared to lower mass stars. They were able to reproduce this gap in their Monte Carlo simulations of planet migration, and they attribute the gap to the shorter depletion timescales of disks around intermediate-mass stars.

The semimajor axis distribution of planets as a function of stellar mass can be investigated further with the inclusion of a larger sample of intermediate-mass subgiants in Doppler-based planet searches. As Figure 9 shows, Doppler surveys of subgiants can probe the occurrence of Jovian planets at orbital distances ranging from many $\mathrm{AU}$ down to as close as $0.05 \mathrm{AU}$, the realm of hot Jupiters. The smaller radii of subgiants also result in higher surface gravities compared to giants, which leads to lower levels of pulsation-induced jitter. Hekker et al. (2006) show that giants with $B-V>1.2$ typically have jitter values greater than $20 \mathrm{~m} \mathrm{~s}^{-1}$, ostensibly due to radial and nonradial pulsation modes. Only giants blueward of this limit are stable to within $20 \mathrm{~m} \mathrm{~s}^{-1}$, compared to the $4-6 \mathrm{~m} \mathrm{~s}^{-1}$ of jitter seen in subgiants $(B-V<1.0$, $M_{V} \lesssim 2.0$ ). This increased velocity stability, coupled with their relatively small radii, therefore make subgiants ideal proxies for A-type dwarfs in Doppler-based planet searches.

The primary limitation of subgiants is their relative scarcity, which restricts the number of bright targets suitable for highresolution spectroscopic observations. The time it takes stars to cross the Hertzsprung gap is small compared to the star's lifetime - of order $100 \mathrm{Myr}$ - rendering Hertzsprung Gap stars within $200 \mathrm{pc}$ rare compared to main-sequence stars and giants. Additional targets can be found further from the Sun, with fainter apparent magnitudes $(V \gtrsim 7.5)$. In the near future, we plan to expand our sample of subgiants using the Keck Telescope and HIRES spectrometer in order to further investigate the orbital properties, planet masses, and occurrence rate of planets orbiting intermediate-mass stars. As the number of subgiants included in Doppler surveys increases, it will become apparent whether the lack of short-period planets around intermediate-mass stars is a result of different formation and migration mechanisms in the disks of A-type stars, or simply a consequence of the small number of massive subgiants currently surveyed.

We extend our gratitude to the many CAT observers who have helped with this project, including Howard Isaacson, Julia Kregenow, Karin Sandstrom, Bernie Walp, Peter Williams, Katie Peek, and Shannon Patel. Special thanks to Hervé Bouy and Francisco Ramos-Stierle for lending a portion of their $3 \mathrm{~m}$ observing time to observe HD 192699 before it set in 2006. We thank Michael Fitzgerald and Marshall Perrin for their useful discussions, and Tim Robishaw for sharing his data display expertise and IDL plotting routines. We also gratefully acknowledge the efforts and dedication of the Lick Observatory and Keck Observatory staff, and the time assignment committees of NASA, $\mathrm{NOAO}$, and University of California for their generous allocations of observing time. We appreciate funding from NASA grant NNG05GK92G (to G. W. M.), and the NSF for its grant AST 03-07493 (to S. S. V.) for supporting this research. D. A. F. is a Cottrell Science Scholar of Research Corporation and acknowledges support from NASA Grant NNG05G164G that made this work possible. This research has made use of the Simbad database operated at CDS, Strasbourg France, and the NASA ADS database. The authors wish to extend special thanks to those of Hawaiian ancestry on whose sacred mountain of Mauna Kea we are privileged to be guests. Without their generous hospitality, the Keck observations presented herein would not have been possible.
Aumann, H. H., et al. 1984, ApJ, 278, L23

Burkert, A., \& Ida, S. 2007, ApJ, 660, 845

Butler, R. P., Marcy, G. W., Williams, E., McCarthy, C., Dosanjh, P., \& Vogt, S. S. 1996, PASP, 108, 500

Butler, R. P., et al. 2006, ApJ, 646, 505

do Nascimento, J. D., Charbonnel, C., Lèbre, A., de Laverny, P., \& De Medeiros, J. R. 2000, A\&A, 357, 931

Flower, P. J. 1996, ApJ, 469, 355

Frink, S., Mitchell, D. S., Quirrenbach, A., Fischer, D. A., Marcy, G. W., \& Butler, R. P. 2002, ApJ, 576, 478

Galland, F., Lagrange, A.-M., Udry, S., Beuzit, J.-L., Pepe, F., \& Mayor, M. 2006, A\&A, 452, 709

Galland, F., Lagrange, A.-M., Udry, S., Chelli, A., Pepe, F., Queloz, D., Beuzit, J.-L., \& Mayor, M. 2005, A\&A, 443, 337

Girardi, L., Bertelli, G., Bressan, A., Chiosi, C., Groenewegen, M. A. T., Marigo, P., Salasnich, B., \& Weiss, A. 2002, A\&A, 391, 195

Gray, D. F., \& Nagar, P. 1985, ApJ, 298, 756

Hatzes, A. P., Cochran, W. D., Endl, M., McArthur, B., Paulson, D. B., Walker, G. A. H., Campbell, B., \& Yang, S. 2003, ApJ, 599, 1383

Hatzes, A. P., Guenther, E. W., Endl, M., Cochran, W. D., Döllinger, M. P., \& Bedalov, A. 2005, A\&A, 437, 743

Hatzes, A. P., et al. 2006, A\&A, 457, 335

Hekker, S., Reffert, S., Quirrenbach, A., Mitchell, D. S., Fischer, D. A., Marcy, G. W., \& Butler, R. P. 2006, A\&A, 454, 943

Johnson, J. A., Marcy, G. W., Fischer, D. A., Henry, G. W., Wright, J. T., Isaacson, H., \& McCarthy, C. 2006a, ApJ, 652, 1724

Johnson, J. A., et al. 2006b, ApJ, 647, 600

Kalas, P., Graham, J. R., \& Clampin, M. 2005, Nature, 435, 1067

Kalas, P., Graham, J. R., Clampin, M. C., \& Fitzgerald, M. P. 2006, ApJ, 637, L57

\section{REFERENCES}

Korzennik, S. G., Brown, T. M., Fischer, D. A., Nisenson, P., \& Noyes, R. W. 2000, ApJ, 533, L147

Lovis, C., Mayor, M., \& Udry, S. 2005, in Proceedings of the 13th Cambridge Workshop on Cool Stars, Stellar Systems and the Sun, ed. F. Favata et al. (ESA SP-560; Nordwijk: ESA), 783

Marcy, G. W., \& Butler, R. P. 1992, PASP, 104, 270

Marcy, G. W., Butler, R. P., Vogt, S. S., Fischer, D. A., Henry, G. W., Laughlin, G., Wright, J. T., \& Johnson, J. A. 2005, ApJ, 619, 570

Maxted, P. F. L., Napiwotzki, R., Dobbie, P. D., \& Burleigh, M. R. 2006, Nature, 442, 543

Neuhäuser, R., Mugrauer, M., Fukagawa, M., Torres, G., \& Schmidt, T. 2007, A\&A, 462, 777

Perrin, M. D., Duchêne, G., Kalas, P., \& Graham, J. R. 2006, ApJ, 645, 1272 Press, W. H., Flannery, B. P., Teukolsky, S. A., \& Vetterling, W. T. 1992, Numerical Recipes: The Art of Scientific Computing (2nd ed.; Cambridge: Cambridge Univ. Press)

Reffert, S., Quirrenbach, A., Mitchell, D. S., Albrecht, S., Hekker, S., Fischer, D. A., Marcy, G. W., \& Butler, R. P. 2006, ApJ, 652, 661

Saar, S. H., Butler, R. P., \& Marcy, G. W. 1998, ApJ, 498, L153

Sato, B., et al. 2003, ApJ, 597, L157 2007, ApJ, 651, 527

Schneider, G., et al. 2006, ApJ, 650, 414

Schrijver, C. J., \& Pols, O. R. 1993, A\&A, 278, 51

Schuler, S. C., Kim, J. H., Tinker, Jr., M. C., King, J. R., Hatzes, A. P., \& Guenther, E. W. 2005, ApJ, 632, L131

Setiawan, J., et al. 2003, A\&A, 398, L19 2005, A\&A, 437, L31

Siess, L., \& Livio, M. 1999, MNRAS, 304, 925

Smith, B. A., \& Terrile, R. J. 1984, Science, 226, 1421 
Takeda, G., Ford, E. B., Sills, A., Rasio, F. A., Fischer, D. A., \& Valenti, J. A. 2007, ApJS, 168, 297

Valenti, J. A., \& Fischer, D. A. 2005, ApJS, 159, 141

Vogt, S. S. 1987, PASP, 99, 1214

Vogt, S. S., et al. 1994, Proc. SPIE, 2198, 362
Wahhaj, Z., Koerner, D. W., \& Sargent, A. I. 2007, ApJ, 661, 368

Wright, J. T. 2004, AJ, 128, 1273 2005, PASP, 117, 657

Wright, J. T., Marcy, G. W., Butler, R. P., \& Vogt, S. S. 2004, ApJS, 152, 261 Wright, J. T., et al. 2007, ApJ, 657, 533 